\title{
Focal Thalamic and Cortical Magnetic Resonance Changes Associated With Partial Status Epilepticus
}

\author{
Franklin Nwokea, b, Yuebing $\mathrm{Li}^{\mathrm{a}, \mathrm{c}}$
}

\begin{abstract}
Status epilepticus (SE) is a commonly occurring life-threatening neurological emergency. Magnetic resonance imaging (MRI) may provide insight into the pathophysiological processes implicated in SE and unique MRI patterns associated with partial SE have recently been reported. We describe transient signal changes on MRI in the areas of cortex and thalamus both ipsilateral to the ictal discharges on electroencephalogram (EEG) in a selected group of five patients with partial SE. We believe this pattern is specific to the peri-ictal state and its recognition is important in those cases where the diagnosis of SE is uncertain. We discuss other common MRI findings seen in SE and possible reasons for similar patterns of corticothalamic signal changes in patients with varying etiologies for SE. The determination of the sensitivity and specificity of such an imaging finding for partial SE still requires further large-scale studies, but a correct recognition of these often reversible imaging abnormalities may help to avoid unnecessary diagnostic tests and invasive procedures.
\end{abstract}

Keywords: Partial status epilepticus; Magnetic resonance imaging; Thalamus

\section{Introduction}

Status epilepticus (SE) is a common life-threatening neuro-

\footnotetext{
Manuscript accepted for publication October 17, 2011

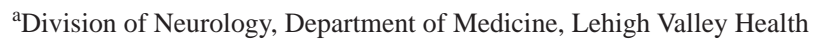
Network, USA

${ }^{\mathrm{b}}$ Diagnostic Radiology, Department of Radiology, Beth Israel Medical Center, First Avenue at 16th Street, New York, NY 10003, USA

${ }^{\mathrm{c} C}$ Corresponding author: Yuebing Li, Email: yuebing.li@lvhn.org
}

doi:10.4021/jnr69w logical emergency. It is defined as continuous seizure activity lasting longer than 30 minutes or sequential seizures without a full recovery of consciousness in between [1]. Magnetic resonance imaging (MRI) is now frequently performed on patients with SE for various reasons. Most commonly it is used for identifying possible underlying structural etiologies and excluding other neurological disorders. In addition, MRI characteristics may provide insight into the pathophysiological processes implicated in SE. For example, vasogenic or cytotoxic cerebral edema has been observed in patients with $\mathrm{SE}$, suggesting increased blood-brain permeability or hypoxia as possible mechanisms of brain injury [2-4]. Finally, unique MRI patterns associated with partial SE have recently been reported [5, 6]. Recognizing these imaging findings as postictal phenomena is valuable in diagnosing and managing SE, especially the ones with atypical clinical presentations such as minimally convulsive or nonconvulsive SE. Here we describe signal changes on MRI in the areas of cortex and thalamus both ipsilateral to the ictal discharges on electroencephalogram (EEG) in a selected group of five patients with partial SE. We believe this pattern is specific to the peri-ictal state and its recognition is important in those cases where the diagnosis of SE is uncertain. We discuss other common MRI findings seen in SE and possible reasons for similar patterns of corticothalamic signal changes in patients with varying etiologies for SE.

\section{Case Report}

\section{Case 1}

A 20-year-old male with a history of mild mental retardation and localization-related epilepsy presented with SE. Seizure episodes began with right arm twitching followed by generalized tonic and clonic convulsions. Complete blood count, electrolyte and spinal fluid analysis were within normal limits. EEG showed continuous 1 to $2 \mathrm{~Hz}$ high-amplitude sharp and slow discharges predominantly over the left frontotemporal region. Multiple medications including pentobarbital infusion were started to terminate SE. MRI on day 3 of admission showed a restricted diffusion in the left 


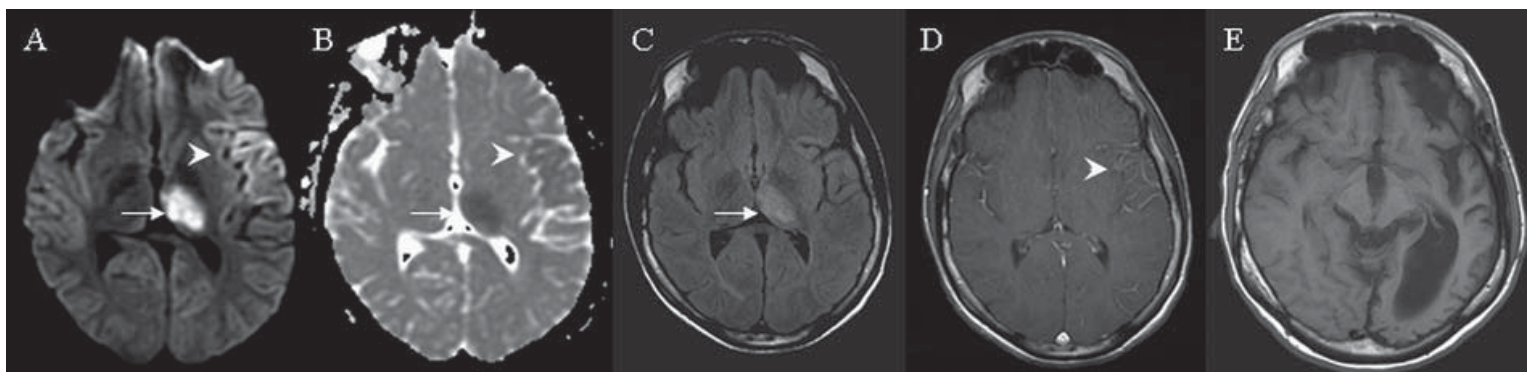

Figure 1. MRI brain findings on case 1. Arrows indicate signal abnormalities at the thalamus. Arrowheads indicate signal abnormalities at the cortex. On day 3 hyperintense signals were seen in the entire left thalamus and gyriform hyperintensities were seen at the left frontotemporal area on diffusion weighted image (A) with corresponding hypointensities on apparent diffusion coefficient (B). Subtle hyperintensities in the left thalamus was seen on fluid attenuated inversion recovery (C) and subtle leptomeningeal enhancement was observed in the left frontotemporal area on T1 weighted image with contrast (D). At nine months T1 weighted image indicated significant left hemispheric atrophy $(E)$.

frontotemporal cortices and the left thalamus. Gyriform cortical hyperintensities were seen on diffusion-weighted images (DWI) corresponding to areas of hypointense signals on Apparent Diffusion Coefficient (ADC). Similar signal changes were observed in the entire left thalamus. Fluid Attenuated Inversion Recovery (FLAIR) images showed hyperintense signals in the left thalamus but without obvious abnormalities in the left frontotemporal cortex. Slight leptomeningeal contrast enhancement was noted in the left frontotemporal region (Fig. 1). The use of pentobarbital infusion led to a burst suppression pattern on the continuous EEG monitoring. A repeat MRI on day 10 showed a complete resolution of signal abnormalities in the left thalamus and left cortical regions on DWI, but subtle hyperintense signals remained within the left thalamus on FLAIR. Upon the discontinuation of pentobarbital, patient continued to have refractory and frequent seizures originating from the left frontotemporal cortex and he eventually underwent a left frontal lobectomy for seizure control. A follow up MRI in nine months demonstrated an extensive volume loss on T1 weighted images and patchy hyperintensities on FLAIR involving the left hemisphere (Fig. 1).

\section{Case 2}

A 27-year-old female with a history of neurosarcoidosis and prior seizures presented with recurrent episodes of confusion, aphasia, right arm paresthesia and weakness. EEG was consistent with non-convulsive focal SE originating from the left temporal lobe spreading to the left parietal cortex. Initial DWI and FLAIR on day 1 showed hyperintensities in the cortical areas surrounding the left central sulcus with corresponding hypointensities on ADC. Repeat DWI and FLAIR images on day 14 showed hyperintensities in the left posterior thalamus (pulvinar) as well as the above cortical areas, corresponding to subtle hypointensities on ADC (Fig. 2). Slight leptomeningeal enhancement was noticed over the left parietal region. Multiple antiepileptic medications including a midazolam drip were given to terminate seizures. A repeat MRI in six weeks showed interval resolution of above diffusion abnormalities with persistent hyperintensities in the left frontal and parietal cortices on FLAIR. Within the next two years, SE originating from the same area recurred and the patient was frequently admitted for seizure control. Repeat MRI of brain in two years revealed a diffuse atrophy of the left hemisphere (Fig. 2).

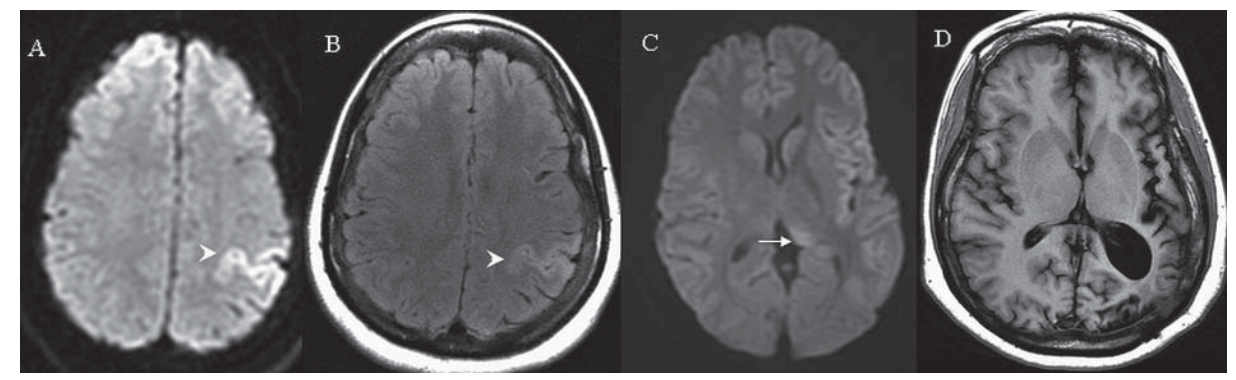

Figure 2. MRI brain findings on case 2. Arrows indicate signal abnormalities at the thalamus. Arrowheads indicate signal abnormalities at the cortical region. On day 1 hyperintensities in the left frontal and parietal cortex were seen on diffusion weighted image (A) and fluid attenuated inversion recovery (B). On day 14, subtle hyperintensities were seen at the left posterior thalamus on diffusion weight image (C). At two years T1 weighted image indicated significant left hemispheric atrophy (D). 


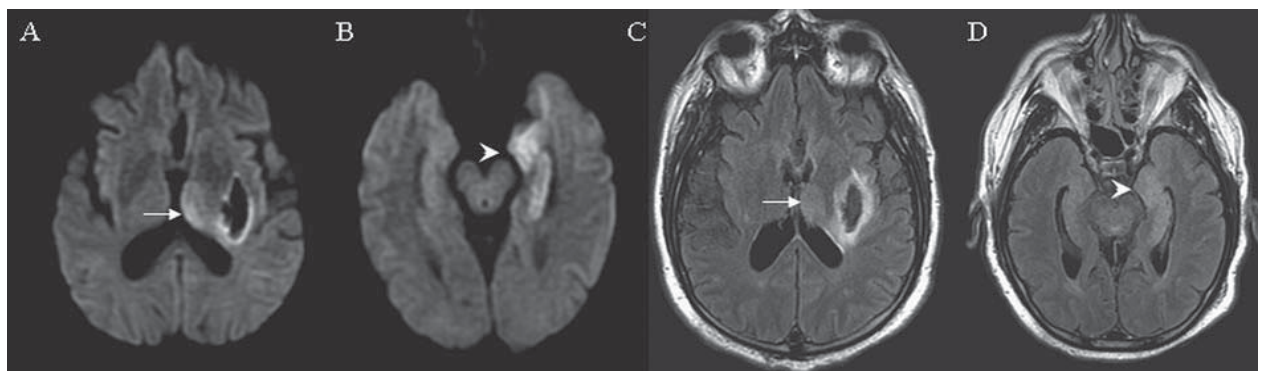

Figure 3. MRI findings on case 3. Arrows indicate signal abnormalities at the thalamus. Arrowheads indicate signal abnormalities at the cortical region. On day 3 diffusion weight image showed hyperintensities in the left thalamus (A) and left medial temporal lobe (B). Similar hyperintensities were seen on FLAIR in the left thalamus (C) and left medial temporal lobe (D). Signal changes in the left lenticular nucleus represent an acute hemorrhage $(A, C)$.

\section{Case 3}

A 60-year-old male with a remote history of alcohol abuse and localization-related epilepsy presented after a witnessed generalized tonic clonic seizure. Intermittent and frequent myoclonic jerks in the right leg and right arm with decreased responsiveness was noticed at admission. CT scans of the head showed an acute hemorrhage in the area of left posterior lenticular nucleus. EEG revealed multiple ictal episodes originating from the left mid-temporal region. MRI on day 3 showed the aforementioned hemorrhage as well as hyperintense lesions in the left medial temporal lobe and left posterior thalamus on both DWI and FLAIR without significant signal abnormalities on ADC (Fig. 3). No contrast enhancement was observed. The patient was treated with multiple antiepileptic medications including propofol which eventually controlled his seizures. No follow up MRI was performed.

\section{Case 4}

A 52-year-old male was admitted with pneumonia and fungal septicemia. On day 1 he developed nine witnessed seizures within a period of two hours. Seizures were characterized by a lack of responsiveness and the presence of tonic/clonic arm movement. EEG showed seizures originating from left or right parietooccipital cortex independently. DWI and FLAIR on day 3 demonstrated diffuse hyperintensities in the bilateral thalami, hippocampi and posterior parietal cortices with corresponding hypointense lesions on ADC (Fig. 4). No contrast enhancement was observed. The patient did not survive the admission and a follow up MRI was not performed.

\section{Case 5}

A 61-year-old male presented with prolonged confusion and left arm automatism. EEG revealed recurrent seizures originating from the right temporal lobe. DWI and FLAIR on day 2 showed subtle hyperintensities in the right hippocampus and right thalamus without corresponding signal changes on ADC (Fig. 4). No contrast enhancement was seen. With treatment, subsequent EEGs did not show recurrent seizure activity. Repeat MRI in eight weeks revealed a resolution of above hyperintense lesions.

\section{Discussion}

We report five cases of partial SE with varying etiologies for seizures. Three of the five patients had prior diagnoses of epilepsy while the remaining two had no such history. All pa-

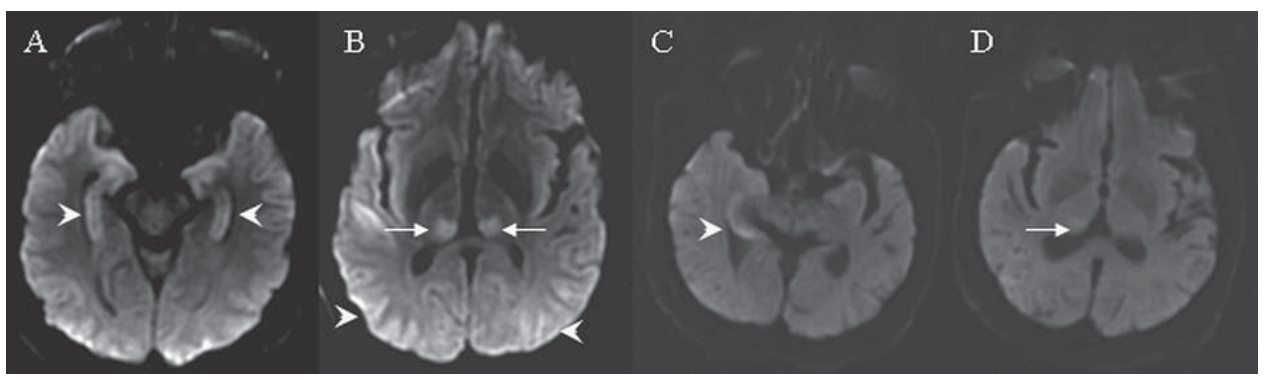

Figure 4. MRI findings on cases 4 and 5. Arrows indicate signal abnormalities at the thalamus. Arrowheads indicate signal abnormalities at the cortical region. In case 4, diffusion weight images showed hyperintensities in the bilateral hippocampus (A), bilateral parietooccipital cortex and bilateral posteior thalamus (B). In case 5, diffusion weighted images showed hyperintensities in the right medial temporal lobe (C) and right posterior thalamus (D). 
tients were placed on continuous EEG monitoring within the first 24 hours of seizure onset. On EEG the origin of SE was unilateral in four and bilaterally independent in the remaining patient. Timing of first MRI scanning varied from within the first 24 hours of SE onset to up to three days after. All five patients had the combination of ipsilateral cortical and thalamic hyperintensities on DWI. The cortical signal abnormalities were irregular and gyriform. The pulvinar area was involved in four of the five patients and the entire thalamus was affected in the remaining patient. FLAIR abnormalities were seen in the same regions as DWI in all five patients but overall more obscure. In all cases DWI and FLAIR abnormalities were ipsilateral to the ictal discharges on EEG and the cortical signal changes corresponded closely in location to the ictal foci. In three patients who had follow up MRI scans, there was a complete resolution of DWI abnormalities as early as ten days after seizure occurrence. In two patients MRI scans at long-term follow up showed a significant parenchymal volume loss ipsilateral to ictal discharges, and in both patients intractable seizures recurred after the initial MRI.

Transient focal MRI changes following partial SE were firstly described by Kramer, et al. [7]. With recent advances in imaging techniques, various findings have been described. Hyperintense lesions in cortical laminar, subcortical or mixed regions have been demonstrated on various imaging modalities that include T2, FLAIR and DWI. The cortical involvement may include temporal, occipital, parietal, insular or frontal lobes, and these signal changes are highly concordant with the ictal discharges on EEG [5, 6, 8-10]. Restricted diffusion on DWI and ADC may mimic acute ischemic stroke, however, these lesions do not respect vascular territories and there are no associated major arterial diseases [6, 11]. Gyral thickening with an obliteration of cortical sulci has been observed indicating cortical edema [12]. The involvement of subcortical white matter is usually limited. Signal abnormalities in the hippocampus, basal ganglia and thalamus are often ipsilateral to the cortical lesions and the ictal discharges $[5,6,10,13]$. In most patients there is a lack of contrast enhancement except minimal leptomeningeal enhancement seen in a minority of cases [11]. Perfusion scans usually reveal cerebral hyperperfusion in the ictal cortex $[5,14]$. Such a regional hyperfusion serves as a compensatory mechanism which is insufficient to avoid hypoxia following prolonged ictal activity.

Very similar patterns of acute MRI findings were detected in SE patients with heterogeneous causes of seizures. The absence of infectious or vascular disorders, the ipsilateral distribution of focal signal abnormalities to the epileptogenic focus, and the disappearance of signal abnormalities following the seizure control all indicate that these acute lesions on MRI are consequences of prolonged epileptic activity rather than the cause of seizures. Such transient T2 signal changes represent focal cerebral edema. Depending on whether the edema is cytotoxic or vasogenic, the predominant diffusion abnormalities in the epileptogenic area may result in a reduction or an increase in ADC or both. The majority of MRI changes after SE resolve within weeks, supporting a mechanism of vasogenic edema due to increased cerebrovascular permeability [5, 13]. Atrophy and persistent T2 hyperintensities were also reported on follow-up MRI scans, suggesting a component of cytotoxic edema and neuronal death [6, $15,16]$. Seizure activity of longer duration is more likely to result in cytotoxic edema, and there is a significant association between the total convulsive time and focal signal changes on imaging $[14,16]$. Sequential seizures can lead to intracellular influx of sodium secondary to the failure of sodium-potassium-ATPases, resulting in subsequent cytotoxic edema. Excessive release of excitatory amino acids, such as glutamate may contribute to edema, necrosis or apoptosis. Three patients in our series demonstrated the presence of cytotoxic edema with DWI hyperintensities and corresponding hypointensities on ADC while the remaining two did not exhibit any noticeable changes on ADC. In the two cases with resultant cerebral atrophy, very prolonged and recurrent seizures were recorded.

Lansberg and colleagues firstly reported postictal T2 hyperintensity in the pulvinar area in a patient with partial status epilepticus [11]. Similar findings have been observed in patients with frontal, temporal, parietal or occipital lobe seizures [5, 6, 9, 12,]. In one prospective case series, pulvinar hyperintensities on DWI were seen in 9 of 10 patients with complex partial SE who underwent serial MR imaging [5]. Katramados et al. reported that among a group of 36 patients with documented clinical and/or electrographic partial SE who underwent peri-ictal MRI, 11 (31\%) had acute thalamic lesions on DWI. In almost all patients, both thalamic and cortical signal abnormalities were ipsilateral to the seizure foci. These thalamic lesions were mostly seen in patients with epileptiform discharges of posterior (temporal, parietal and occipital) origin, and the cortical involvement appeared to derive mainly from the dorsolateral surfaces rather than a midsagittal location [6]. The thalamus may serve as a relay point in the pathway of seizure propagation. In rat models, thalamic epileptiform activities appeared simultaneously with those in the cortical focus, and the intensity of epileptic activity in thalamus increased in parallel with that in the cortical focus as well [17]. Primate models of absence SE have demonstrated an activation of the corticothalamic circuitry that corresponds to epileptiform discharges [18]. Using intracerebral EEG recording, Guye et al. demonstrated synchronized electrical activity between thalamus and temporal lobe structures during temporal lobe seizures [19].

While pulvinar hyperintensity is the most commonly reported MRI abnormality, an involvement of other areas of thalamus has been identified such as the entire thalamus [13]. Case No. 1 in our series also revealed such a finding on imaging. Similar to cortical signal abnormalities following SE, 
ipsilateral focal thalamic hyperintense lesions are predominantly seen on DWI reflecting acute thalamic dysfunction. Thalamic lesions do not demonstrate contrast enhancement and there is a lack of evidence for vascular, inflammatory or neoplastic disorders affecting the thalamic area [20]. Therefore, thalamic lesions are purely consequences of the SE and not of a preexisting dysfunction. Similar to cortical lesions, persistent signal abnormalities in thalamus and thalamic atrophy can be observed in future stages [20].

\section{Conclusion}

The presence of unilateral gyriform cortical and thalamic hyperintense lesions on MRI, especially on DWI, is a unique and distinct image finding in complex partial SE. To our knowledge, such a combination has not been reported in other acute neurological disorders. The determination of the sensitivity and specificity of such an imaging finding for partial SE still requires further large-scale studies, but the identification of this pattern is particularly useful in cases of non convulsive SE such as in elderly patients with prolonged confusional syndromes. A correct recognition of these often reversible imaging abnormalities may help to avoid unnecessary diagnostic tests and invasive procedures.

\section{References}

1. Treatment of convulsive status epilepticus. Recommendations of the Epilepsy Foundation of America's Working Group on Status Epilepticus. JAMA. 1993;270(7):854859.

2. Meierkord H, Wieshmann U, Niehaus L, Lehmann R. Structural consequences of status epilepticus demonstrated with serial magnetic resonance imaging. Acta Neurol Scand. 1997;96(3):127-132.

3. Amato C, Elia M, Musumeci SA, Bisceglie P, Moschini M. Transient MRI abnormalities associated with partial status epilepticus: a case report. Eur J Radiol. 2001;38(1):50-54.

4. Chu K, Kang DW, Kim JY, Chang KH, Lee SK. Diffusion-weighted magnetic resonance imaging in nonconvulsive status epilepticus. Arch Neurol. 2001;58(6):993998.

5. Szabo K, Poepel A, Pohlmann-Eden B, Hirsch J, Back T, Sedlaczek O, Hennerici M, et al. Diffusion-weighted and perfusion MRI demonstrates parenchymal changes in complex partial status epilepticus. Brain. 2005;128(Pt 6):1369-1376.

6. Katramados AM, Burdette D, Patel SC, Schultz LR, Gaddam S, Mitsias PD. Periictal diffusion abnormalities of the thalamus in partial status epilepticus. Epilepsia.
2009;50(2):265-275.

7. Kramer RE, Luders H, Lesser RP, Weinstein MR, Dinner DS, Morris HH, Wyllie E. Transient focal abnormalities of neuroimaging studies during focal status epilepticus. Epilepsia. 1987;28(5):528-532.

8. Diehl B, Najm I, Ruggieri P, Tkach J, Mohamed A, Morris $\mathrm{H}$, Wyllie E, et al. Postictal diffusion-weighted imaging for the localization of focal epileptic areas in temporal lobe epilepsy. Epilepsia. 2001;42(1):21-28.

9. Di Bonaventura C, Bonini F, Fattouch J, Mari F, Petrucci S, Carni M, Tinelli E, et al. Diffusion-weighted magnetic resonance imaging in patients with partial status epilepticus. Epilepsia. 2009;50 Suppl 1:45-52.

10. Gujjar A, Jacob PC, Al-Asmi A, Ramachandiran N, Obaidi A, Jain R. Reversible MRI changes in prolonged status epilepticus: a case report. Int J Neurosci. 2011;121(6):341-345.

11. Lansberg MG, O’Brien MW, Norbash AM, Moseley ME, Morrell M, Albers GW. MRI abnormalities associated with partial status epilepticus. Neurology. 1999;52(5):1021-1027.

12. Nagasaka T, Shindo K, Hiraide M, Sugimoto T, Shiozawa Z. Ipsilateral thalamic MRI abnormality in an epilepsy patient. Neurology. 2002;58(4):641-644.

13. Huang YC, Weng HH, Tsai YT, Hsiao MC, Wu CY, Lin $\mathrm{YH}$, Hsu HL, et al. Periictal magnetic resonance imaging in status epilepticus. Epilepsy Res. 2009;86(1):7281.

14. Cole AJ. Status epilepticus and periictal imaging. Epilepsia. 2004;45 Suppl 4:72-77.

15. Hong KS, Cho YJ, Lee SK, Jeong SW, Kim WK, Oh EJ. Diffusion changes suggesting predominant vasogenic oedema during partial status epilepticus. Seizure. 2004;13(5):317-321.

16. Goyal MK, Sinha S, Ravishankar S, Shivshankar JJ. Peri-ictal signal changes in seven patients with status epilepticus: interesting MRI observations. Neuroradiology. 2009;51(3):151-161.

17. Sharma V, Singh R. Thalamic neuropathology in the chronic pilocarpine and picrotoxin model of epilepsy. Indian J Exp Biol. 1999;37:461-467.

18. Tenney JR, Marshall PC, King JA, Ferris CF. fMRI of generalized absence status epilepticus in conscious marmoset monkeys reveals corticothalamic activation. Epilepsia. 2004;45(10):1240-1247.

19. Guye M, Regis J, Tamura M, Wendling F, McGonigal A, Chauvel P, Bartolomei F. The role of corticothalamic coupling in human temporal lobe epilepsy. Brain. 2006;129(Pt 7):1917-1928.

20. Tschampa HJ, Greschus S, Sassen R, Bien CG, Urbach $\mathrm{H}$. Thalamus lesions in chronic and acute seizure disorders. Neuroradiology. 2011;53(4):245-254. 